\title{
Ecuador del boom a la crisis: Política fiscal para postergar el ajuste
}

\author{
Nicolás Acosta-González', Sebastián Padilla-Tapia²
}

Fecha de recepción: 25 de Abril 2018

\section{Resumen}

La emisión de deuda se convirtió en una importante fuente de ingresos del Ecuador a partir de 2016. El país venía experimentado una complicada situación fiscal desde el 2013 la cual se agravó cuando el precio del petróleo se desplomó y un terremoto de 7,8 grados golpeó el litoral. En este trabajo, mediante un análisis correlacional de las variables se emiten explicaciones e hipótesis acerca de la sostenibilidad fiscal, la calidad del gasto, y algunos instrumentos de financiamiento no convencionales. Se concluye que, a pesar del uso de estos instrumentos con el fin de postergar el ajuste, la situación fiscal es ya insostenible.

Palabras clave: Fiscal, sostenibilidad, blanchard, ajuste, devaluación

\section{Abstract}

Debt issuance was an important source of income for Ecuador since 2016. The country was already facing a complicated fiscal scenario since 2013. When the oil price collapsed and an earthquake of 7,8 magnitude hit the coast, fiscal balance got highly deteriorated. In this paper, through correlational treatment of the variables we issue explanations and hypothesis about fiscal sustainability, quality of spending, and some unconventional instruments. We conclude that, despite the use of these instruments to postpone a fiscal adjustment, the situation is already unsustainable.

Keywords: Fiscal, sustainability, blanchard, adjustment, devaluation

\section{Introducción}

El Ecuador, país dolarizado y exportador de petróleo, fue beneficiario del boom internacional de precios de los commodities desde el $2007 \mathrm{al}$ 2015. Durante este periodo, el motor de la economía ecuatoriana fue el gasto público, tanto corriente como el de inversiones en infraestructura, el cual creció de manera desproporcional en relación a los ingresos. Tal fue la magnitud de los gastos realizados que, en 2013 en pleno boom, con precios de petróleo mayores a $\$ 90$ dólares por barril, el déficit fiscal se encontraba ya en -5,74\% del PIB; mientras el déficit se ha mantenido alrededor de esa cifra hasta 2017 (-5,96\% PIB), la deuda pública pasó del $23 \%$ al $46 \%$ del PIB en el mismo periodo.

La emisión de deuda se convirtió en la fuente de ingresos de un país con una situación fiscal complicada que se agravó dos años más tarde cuando a finales del 2015 el precio del petróleo se desplomó llegando a cotizarse por debajo de $\$ 25$ por barril sumado a que, en 2016, dos provincias ecuatorianas sufrieron un terremoto de 7,8 grados en la escala de Richter que dejó un saldo de reconstrucción pendiente de \$3.344 millones.

Bajo estas circunstancias, el ajuste fiscal es necesario para estabilizar las finanzas públicas ya que la evidencia muestra beneficios en el largo plazo. En este sentido, el reporte semianual de la oficina del Economista en Jefe del Banco Mundial para América Latina y el Caribe publicado en abril del 2018, cuantifica los efectos en el largo plazo de un ajuste fiscal, específicamente se observa que una reducción de un punto porcentual en el déficit primario se traduce en un aumento de 0,2 puntos porcentuales en el crecimiento en el largo plazo (Végh, y otros, 2018). A grandes rasgos, un ajuste fiscal no ha llegado a producirse, aunque en los últimos meses se habla de un ajuste gradual. En ese sentido, en un país sin la capacidad de emisión monetaria, el análisis de ciertos tópicos de la política fiscal para hacer frente a los problemas descritos resulta preponderante.
Fecha de aceptación: 22 de Junio 2018

En primer lugar, se analizará los ingresos provenientes de deuda y la sostenibilidad fiscal, en segundo lugar, un breve apartado sobre la calidad del gasto público, y en tercer lugar el controvertido financiamiento con fondos del Banco Central y una iniciativa innovadora que fue planteada pero que no llegó a realizarse conocida como devaluación fiscal.

\section{Metodología}

En el presente artículo se realiza un análisis descriptivo de las variables que configuran el sector fiscal y a través de las cuales se establecen relaciones de concomitancia que no buscan determinar efectos causales. A partir del trato correlacional a las variables se emiten explicaciones e hipótesis acerca de los temas tratados. Se utiliza como fuente de datos las publicaciones oficiales del Banco Central del Ecuador tanto de las cuentas trimestrales como de la Información Estadística Mensual y los datos de deuda pública publicados por el Ministerio de Economía y Finanzas. Esta información ha sido recopilada y sistematizada por el equipo del Reporte de Consistencia Macroeconómica-RCM de la Pontificia Universidad Católica del Ecuador-PUCE.

\section{Resultados}

\section{Deuda y sostenibilidad fiscal}

Aunque es evidente que adquirir deuda para el financiamiento público es una práctica necesaria, ya que puede ser usada para incrementar la productividad o el capital a través de inversiones puntuales, también puede convertirse en una carga a futuro para el Estado. En este sentido se debe analizar el stock de deuda, el déficit fiscal, las fuentes de financiamiento, las tasas de interés, los plazos y la tasa de crecimiento para obtener una idea clara de la sostenibilidad fiscal. El indicador de Blanchard (1990) recoge las condiciones descritas y es el referente mundial para evaluar el desarrollo de las finanzas públicas. En cuanto al stock de deuda, las autoridades económicas del Ecuador se encuentran en conflicto para determinar la fórmula de cálculo y por consiguiente el monto de la deuda pública. El informe DNA3-0007-2018 emitido por la Contraloría General del Estado ratifica observaciones al manejo de deuda principalmente en la no incorporación de las ventas anticipadas de petróleo como deuda y el tratamiento a la información de financiamiento público como secreta y reservada a más del cambio de fórmula.

Dentro del gobierno existen dos perspectivas, la primera desde el Ministerio de Finanzas se habla de deuda consolidada (35\% PIB), la cual resta gran parte de la deuda interna, en su mayoría con el Instituto Ecuatoriano de Seguridad Social-IESS por alrededor de $\$ 14.800$ millones; la segunda parte de declaraciones del Presidente Moreno en la que se reconoce a los pasivos totales como stock de deuda (69\% PIB).

Por otro lado, la deuda agregada que incluye los saldos de deuda externa e interna (48\% PIB), nunca dejó de ser utilizada como medida de sostenibilidad fiscal por parte de bancos multilaterales, universidades y centros de investigación y es la metodología utilizada por el Fondo Monetario Internacional-FMl para presentar sus reportes de deuda bruta (Fondo Monetario Internacional, 2014).

Respecto al déficit fiscal, el año 2017 cerró con un resultado global del Gobierno Central de -\$6.140 millones de dólares (6\% PIB). En ese sentido a inicios de 2017 el Ministerio de Finanzas proyectó necesidades de financiamiento por $\$ 11.670$ millones, sin embargo, el resultado de desembolsos de deuda a finales de año fue superior en más de $\$ 4.000$ millones ubicándose en \$15.797 millones. (Banco Central del Ecuador, 2018)

\footnotetext{
${ }^{1}$ Master of Arts in Economics por Georgetown University. Director del Reporte de Consistencia Macroeconómica-RCM. Profesor de la Facultad de Economía de la Pontificia Universidad Católica del Ecuador. Quito- Ecuador. E-Mail: hnacosta@puce.edu.ec

${ }^{2}$ Egresado de la Facultad de Economía de la Pontificia Universidad Católica del Ecuador. Investigador del Reporte de Consistencia Macroeconómica-RCM. Facultad de Economía de la Pontificia Universidad Católica del Ecuador. Quito- Ecuador. E-Mail: apadilla648@puce.edu.ec
} 
El detalle de tasas de interés, plazos y calificación de riesgo se puede observar en la siguiente tabla:

Tabla 1. Características de emisión de bonos de deuda

\begin{tabular}{|c|c|c|c|c|c|}
\hline & $\begin{array}{l}\text { Fecha } \\
\text { emisión }\end{array}$ & $\begin{array}{l}\text { Monto } \\
\text { USD MM }\end{array}$ & Plazo & Tasa & $\begin{array}{c}\text { Calificación } \\
\left(\mathrm{S} \& \mathrm{P}^{*}\right)\end{array}$ \\
\hline Bonos Soberanos 2024 & Junio 2014 & 2.000 & 10 años & $7,95 \%$ & B \\
\hline Bonos Soberanos 2020 & Marzo 2015 & 1.500 & 5 años & $10,50 \%$ & $\mathrm{~B}+$ \\
\hline Bonos Soberanos 2022 & Julio 2016 & 1.000 & 6 años & $10,75 \%$ & B \\
\hline Bonos Soberanos 2026 & $\begin{array}{c}\text { Diciembre } \\
2016\end{array}$ & 1.000 & 10 años & $9,125 \%$ & B \\
\hline Bonos Soberanos 2023 & Mayo 2017 & 1.000 & 6 años & $8,75 \%$ & B \\
\hline Bonos Soberanos 2027 & Junio 2017 & 1.000 & 10 años & $9,62 \%$ & B \\
\hline Bonos Soberanos 2027 & Octubre 2017 & 2.500 & 10 años & $8,875 \%$ & B- \\
\hline Bonos Soberanos 2028 & Enero 2018 & 3.000 & 10 años & $7,875 \%$ & B- \\
\hline Total y promedio & & 13.000 & 8,3 años & $9,17 \%$ & \\
\hline
\end{tabular}

Fuente: Ministerio de Economía y Finanzas, Boletín de Deuda Externa Nro. 253 (2018): *Standard \& Poor's Financial Services- S\&P, Listado de Calificaciones por Emisor América Latina de SP Global Ratings (2018)

Si bien se evidencia en 2017 y 2018 un incremento en los montos emitidos acompañado de un aumento de plazos y una reducción de la tasa de interés, la mejora en las condiciones financieras no va acompañada de una mejor calificación crediticia de la deuda ecuatoriana y en su lugar disminuye de B a B-. Una posible explicación a esta aparente inconsistencia es que en el esquema de endeudamiento externo el precio de petróleo juega un rol preponderante en el establecimiento del riesgo país con la consecuente afectación a las tasas de interés y plazos.

Mientras que en enero de 2016 el barril de petróleo se vendió en \$31, el riesgo país se ubicó en 1509 puntos y dos años más tarde en enero del 2018 el preció llegó a \$64 y el riesgo país cayó a 453 puntos. Es así como la percepción por parte de inversionistas de que existe una mejora en la confianza parece ser que se circunscribe a factores exógenos más que a medidas de política económica.

Existen varias razones para que tanto la deuda como el déficit fiscal hayan aumentado desde 2014. Por un lado, la disminución del precio de petróleo y la consiguiente apreciación del tipo de cambio del dólar frente a casi todas las monedas tuvo efectos directos en la economía. Esto pudo haber sido consecuencia de problemas coyunturales como la debilidad del sector externo y en el gran papel del gasto público corriente y de capital, que fue canalizado al sector privado a través de obras e infraestructura en general y de problemas estructurales como la poca diversificación de la oferta exportable.

Finalmente, el indicador de Blanchard que se refiere al ajuste en el balance fiscal primario necesario para garantizar la sostenibilidad de la política fiscal cerró en 2017 en 4,8\%, es decir bajo un sistema de política fiscal insostenible.

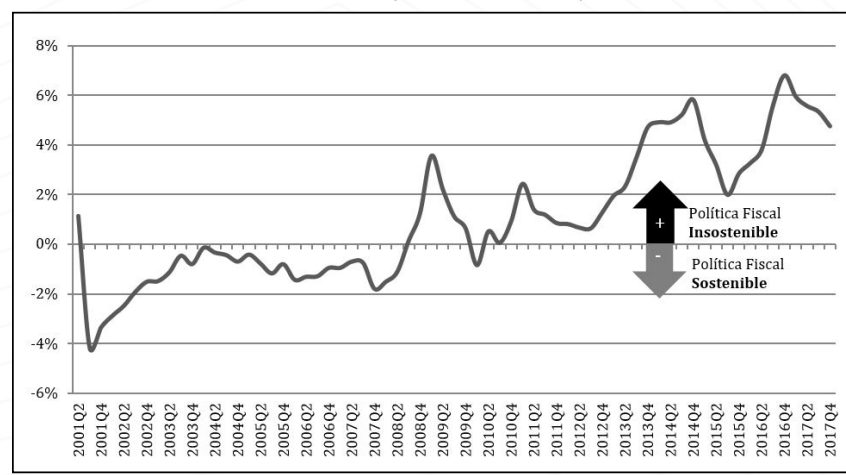

Fuente: (Banco Central del Ecuador-BCE, Información Estadística Mensual 1993, 2018) Figura 1. Indicador de Blanchard del Gobierno Central

\section{Calidad del gasto público}

La calidad del gasto público es de marcado interés para las economías en las que el gasto es un motor de crecimiento económico como es el caso de Ecuador. Debido a que una estimación puntual de la calidad del gasto público en Ecuador resulta complicada y trabajos anteriores han utilizado métodos no paramétricos como por ejemplo Ribeiro (2008), en esta ocasión se analiza aportes teóricos sobre indicadores de calidad del gasto y los resultados de un estudio sobre eficiencia del gasto en inversión pública llevado a cabo por el Banco Interamericano de Desarrollo-BID para toda Latinoamérica.

Dentro de los aportes tanto teóricos como empíricos para ahondar el análisis en política fiscal surge un concepto multidimensional que se conoce como la Calidad de las Finanzas Públicas-CFP, éste hace referencia a los acuerdos y operaciones de política que contribuyen a cumplir objetivos macroeconómicos de política fiscal, principalmente las que impactan en el crecimiento a largo plazo (Barrios \& Schaechter, 2009).

Las cinco dimensiones del concepto son (1) el tamaño del gobierno, (2) la posición fiscal y sostenibilidad, (3) la composición, eficiencia y efectividad del gasto, (4) la estructura y eficiencia de los impuestos y (5) la gobernanza fiscal. Como se puede apreciar, una de las dimensiones para que la política fiscal tenga un impacto en el crecimiento a largo plazo, es la que nos concierne analizar: la composición, eficiencia y efectividad del gasto.

La eficiencia o calidad del gasto público en una economía puede ser evaluado bajo diversas percepciones. Por ejemplo, para la Comisión Económica para América Latina y el Caribe (CEPAL) se trata del uso eficaz y eficiente de los recursos públicos con los objetivos de elevar el potencial de crecimiento de la economía, y en el caso de América Latina, asegurar crecientes grados de equidad distributiva. Por su parte, el BID, de manera más acotada, hace énfasis en el análisis del efecto del gasto en inversión pública en el crecimiento, y las relaciones entre el stock de infraestructura, su calidad y los niveles de ingreso per cápita. Finalmente existe un indicador del Foro Económico Mundial sobre eficiencia del gasto público calculado en base a encuestas a ejecutivos con el fin de obtener el resultado de Institucionalidad como pilar del Reporte Anual de Competitividad Global.

Un aporte para medir la calidad del gasto público es el estudio realizado por Afonso, Schuknecht \& Tanzi (2005) en el que realizaron una aproximación del resultado del desempeño del sector público en 23 países de la Organización para la Cooperación y el Desarrollo Económicos-OCDE entre 1990 y 2000. Se seleccionó una serie de indicadores de los cuales los primeros reflejan los procesos de interacción entre las políticas fiscales y el mercado, y los segundos las tareas del gobierno en desempeño económico, estabilidad y distribución. A continuación, se presenta el detalle:
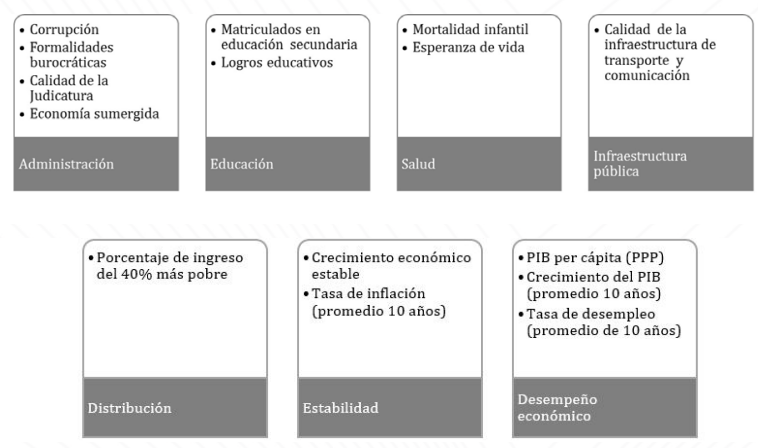

Fuente: Afonso, Schuknetch, \& Tanzi (2005)

Figura 2. Indicadores para evaluar la calidad del gasto público

Por otro lado, para evaluar la calidad del gasto en inversión pública en América Latina, el BID basado en un estudio de Armendáriz \& Contreras (2016) propuso un índice ponderado de eficiencia de la gestión de la inversión pública (0 baja eficiencia- 4 alta eficiencia) que consta de 5 dimensiones: guías estratégicas, selección de proyectos, implementación de proyectos, evaluación de proyectos y caracterización general del ciclo de inversión pública.

En este índice el Ecuador obtiene un puntaje total ponderado de 2,5 sobre 4. Por dimensiones el mayor puntaje corresponde a la dimensión 
selección del proyecto, incluso por encima de la media latinoamericana. El puntaje tiene sentido ya que la mayor inversión estuvo destinada a proyectos de generación eléctrica, carreteras, escuelas y hospitales, los cuales cumplen con el objetivo de elevar el potencial de crecimiento de la economía. En la dimensión sobre guías estratégicas de inversión en la que se encuentra la valoración de precios sociales, el Ecuador obtiene un puntaje por debajo de la media latinoamericana.

Lastimosamente la peor evaluación para el Ecuador es la que tiene que ver con implementación de proyectos con un puntaje de 2, en esta dimensión Ecuador obtiene el peor puntaje de todos los países de Latinoamérica. Esta dimensión del indicador hace referencia a la adjudicación, cumplimiento temporal y auditoría de los proyectos. El bajo puntaje casualmente se relaciona con los problemas de coimas, retrasos en obras y corrupción de los organismos de control que se han investigado desde el 2017 en Ecuador.

\section{Financiamiento con el Banco Central del Ecuador}

El financiamiento del Banco Central del Ecuador-BCE al Gobierno Central ha sido un instrumento utilizado durante gran parte de las últimas décadas, tanto con emisiones inorgánicas cuando existía soberanía monetaria, así como en años recientes con recursos de la Reservas Internacionales-RI bajo un sistema dolarizado.

Las Reservas Internacionales-RI son activos públicos y privados que por disposición legal están en manos del BCE. Aunque las RI no respaldan a la dolarización en su conjunto, la liquidez total a marzo del 2018 es de $\$ 51.155$ millones mientras que las RI llegan a $\$ 4.570$ millones con datos al 6 de abril del 2018, las RI deberían respaldar: (1) la emisión de monedas y dinero electrónico, (2) el encaje bancario, tanto privado como público, y (3) el dinero del sector público no financiero.

¿Por qué deberían respaldar las Rl a estos tres rubros? Sencillamente, porque estas reservas tienen dueño y en su contraparte del balance son pasivos exigibles. En el lado de los activos, las RI como ya se señaló llegan a \$4.570 millones. En el lado de los pasivos se encuentran: la emisión monetaria y dinero electrónico que son parte del sistema de canje y suman $\$ 89,4$ millones; las Reservas Bancarias que suman $\$ 4.570$ millones e incluyen encajes de entidades financieras públicas y privadas; y por último los depósitos del sistema público no financiero que suman $\$ 6.899$ millones.

En la teoría monetaria clásica para un país con moneda propia, sin que el Banco Central actúe como un banco comercial cualquiera, los pasivos exigibles que suman $\$ 11.736$ millones deberían estar respaldados por las RI. Esta situación no sucede en el Ecuador, existiendo una cobertura del 39\%. Si se excluyen los depósitos del sistema público no financiero, el resultado es conocido como "Pasivos Monetarios" y la cobertura es del $94 \%$ de los pasivos, es decir, no se respalda el sistema de canje ni el de reservas bancarias.

Esta falta de cobertura empezó en diciembre del 2015, mes en el que por primera vez los pasivos monetarios fueron mayores a las RI por 644 millones de dólares y se ha mantenido por debajo durante más de dos años, aunque con un par de meses en los que las RI han sido superiores y que coinciden con el ingreso de fondos fruto de colocaciones de bonos de deuda externa, en octubre de 2017 y enero 2018.

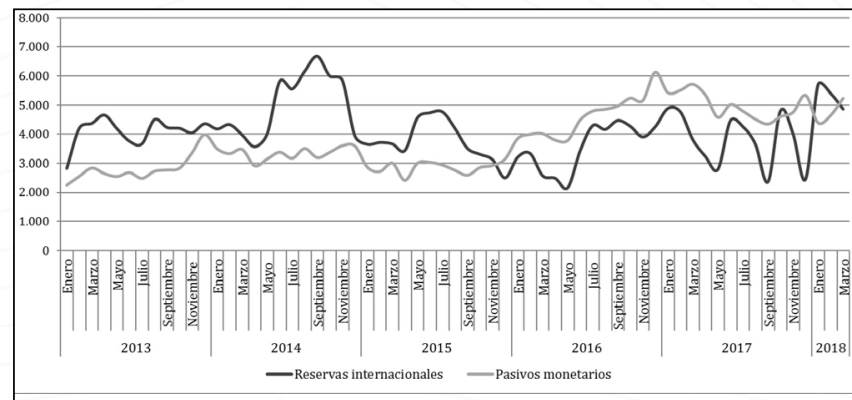

Fuente: (Banco Central del Ecuador-BCE, Boletín Monetario Semanal 583, 2018) Figura 3. Reservas Internacionales
Mientras el BCE registraba en la cuenta "Valores distintos de acciones" préstamos al Gobierno Central, la cual aumentó en \$5,800 millones de 2014 a 2017, las instituciones financieras se enfrentaban en 2017 a una caída de los depósitos que provocó una contracción de la oferta crediticia al microcrédito y segmento de consumo, más una caída de la demanda de crédito corporativo.

Si bien este desbalance toma en cuenta también las reservas bancarias públicas, en 4 meses durante el 2017 (abril, mayo, septiembre y diciembre) una parte de estas reservas que corresponden al encaje bancario privado fue mayor que las RI. Existía la preocupación de que el gobierno esté siendo financiado no sólo con las reservas bancarias totales sino con el encaje bancario privado, sin consentimiento de sus administradores y peor de los depositantes.

\section{Pago de deuda con acciones}

A pesar de las dudas acerca del origen de los fondos para el financiamiento del Gobierno Central por parte del BCE, al menos las obligaciones contraídas habían sido debidamente pagadas en efectivo sin arriesgar en mayor medida la liquidez del BCE. Sin embargo, la publicación por parte del BCE del Boletín Monetario Semanal con fecha 31 de mayo del 2017 correspondiente a la semana que concluyó el 19 de mayo trajo una sorpresa para muchos, se registró en el balance sectorial un incremento, respecto a la semana anterior de \$2.136,6 millones en la cuenta de acciones y otras participaciones de capital. Vale destacar que este boletín se presentó con demora ya que el 25 de mayo se publicó la nueva Metodología de la Información Estadística, en este documento se crea una subcuenta correspondiente a "otras sociedades financieras" la cual no existía en la anterior metodología dentro de la cuenta de acciones.

El 2 de junio del 2017 el Ministerio de Finanzas confirmó que se trataron de acciones de instituciones financieras públicas, un total de $\$ 1.440$ millones de la Corporación Financiera Nacional-CFN, \$453 millones de BanEcuador y \$245 millones de Corporación Nacional de Finanzas Populares y Solidarias-CONAFIPS. El motivo era "diversificar sus activos sin afectar la liquidez y aumentar su rendimiento", la realidad es que al mismo tiempo se registró una disminución de las obligaciones del Estado con el BCE al pasar de \$5.866 a \$3.728 millones.

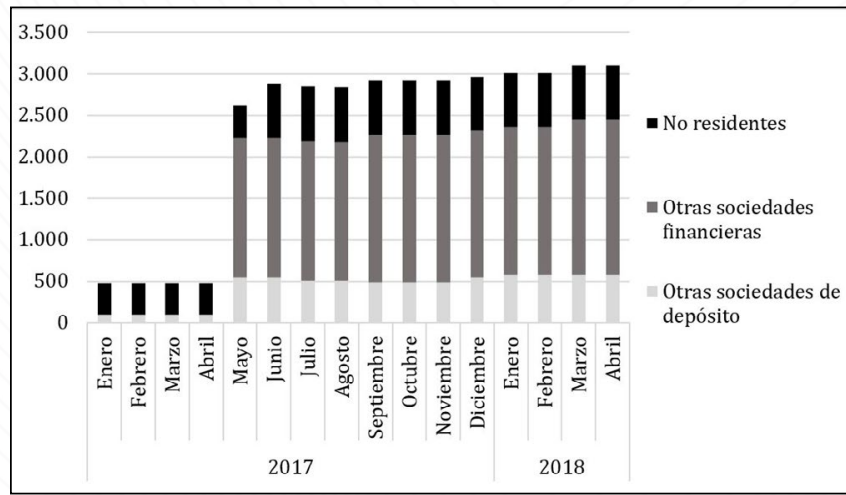

Fuente: (Banco Central del Ecuador-BCE, Boletín Monetario Semanal 583, 2018) Figura 4. Total acciones y otras participaciones de capital

Al 6 de abril de 2018, el BCE registra $\$ 3.099$ millones de acciones y de esta manera ha seguido aumentando sus activos con poca liquidez. En definitiva, el Gobierno Central había pagado una deuda al BCE con acciones de bancos públicos, sin dar a conocer los métodos para valorar las acciones convirtiendo a la transacción en un simple arreglo contable que resta liquidez al BCE porque se incluye en el balance activos muy difíciles de monetizar.

\section{Devaluación Fiscal}

Finalmente, se analiza una medida anunciada pero que nunca llegó a materializarse. Una devaluación fiscal es una medida que busca reducir los costos de producción de las empresas para incrementar su competitividad, la manera más directa de lograrlo es ofrecer un subsidio a las exportaciones y compensarlo con un incremento de tarifas a las 
importaciones para mantener en equilibrio las cuentas fiscales y así lograr un incremento del producto y del nivel de empleo. En todo caso, cualquier reducción de los costos de producción debe ir acompañado de un incremento en los ingresos fiscales.

Existen otras maneras de devaluación fiscal que se han aplicado en los últimos años en países de la Unión Europea como España y Alemania, los cuales enfrentan por un lado una incapacidad de imponer impuestos a las importaciones o restringir el comercio al ser parte de una unión supranacional y por otro lado no pueden acudir devaluaciones monetarias, ésta última situación es similar a la del Ecuador. Las devaluaciones fiscales en Europa se han logrado través de reducir los aportes a la Seguridad Social equilibrándolo con aumento en el Impuesto al Valor Agregado -IVA.

El hecho de haberse aplicado en otros países con anterioridad ha motivado que se realicen estudios sobre el efecto de la devaluación fiscal en el PIB y el empleo. Aunque las condiciones económicas entre el Ecuador y por ejemplo España no se pueden establecer como similares, se presenta los resultados obtenidos para tener una idea general. En ese estudio, Boscá, Doménech y Ferri (2013) estimaron que una disminución de 3,5 puntos en los aportes a la Seguridad Social y un aumento de 2 puntos en el IVA estaban relacionados con un incremento de 200 mil empleos tras dos años y un aumento del 0,74\% del PIB.

Para el Ecuador, las posibilidades para contrarrestar el gasto extra de asumir una parte de los aportes patronales a la Seguridad Social van en la línea de un incremento de impuestos, que pudieron ser el IVA, impuestos ambientales o impuestos a las importaciones de bienes de consumo; todo esto siempre y cuando no afecte a la estructura productiva de las empresas ni su tasa de ganancia, ya que es justamente al sector al que se intenta ayudar con el fin de incrementar las exportaciones; caso contrario el incremento de los costos sería asumido por la ciudadanía de una u otra manera. Otra posibilidad sería sumar el porcentaje de aporte patronal a la deuda ya existente con el Instituto de Seguridad Social y de esa manera obtener los beneficios de la devaluación sin que la ciudadanía asuma los costos por el momento, eso sí agravando el déficit actuarial de la Seguridad Social. En cualquier caso, los efectos de la devaluación fiscal no difieren mucho de una devaluación del tipo de cambio: se incrementan las exportaciones, crece de manera momentánea el PIB, el empleo y se reduce el déficit de cuenta corriente.

\section{Conclusiones}

Para mantener el modelo económico, las tasas de empleo y el déficit fiscal en torno al 6\% desde el 2013, se postergó el ajuste suplantando el ingreso petrolero con emisión de deuda. En 2017 y 2018, se evidencia un incremento en los montos emitidos y una reducción de la tasa de interés, esta mejora en las condiciones financieras no va acompañada de una mejor calificación crediticia de la deuda ecuatoriana y en su lugar disminuye de B a B-, una posible explicación es que el precio de petróleo juega un rol preponderante en el establecimiento del riesgo país con la consecuente afectación a las tasas de interés y plazos.

La sostenibilidad fiscal es uno de los mayores problemas para las finanzas públicas ecuatorianas, el indicador de Blanchard, que se refiere al ajuste en el balance fiscal primario necesario para garantizar la sostenibilidad de la política fiscal, ha venido en aumento desde el 2010 y cerró en 2017 en 4,8\% que indica un problema de insostenibilidad fiscal. Este índice tuvo sus picos en 2008 y 2016, llegando a 7\%.

Por otra parte, los diferentes aportes teóricos coinciden en que el fin del gasto público es elevar la potencialidad del crecimiento económico y en el caso de América Latina permita mayores grados de equidad distributiva. El análisis del gasto en infraestructura concluye que Ecuador recibe buen puntaje en la dimensión correspondiente a selección de proyectos y el peor puntaje de Latinoamérica en la implementación, cumplimiento y auditoría. Estos resultados van acorde a los problemas que ha surgido respecto a sobreprecios y coimas.

El papel del BCE en el financiamiento al Gobierno genera controversia. En primer lugar, porque existe la preocupación de que el gobierno esté siendo financiado no solo con las reservas bancarias que incluyen a los bancos públicos sino incluso con el encaje bancario privado, sin consentimiento de sus administradores y peor de los depositantes. En segundo lugar, porque el Gobierno Central pagó una parte de sus obligaciones al BCE con acciones de bancos públicos, sin dar a conocer los métodos para valorar las acciones que en definitiva son activos muy difíciles de monetizar afectando la liquidez del BCE. Estas acciones solo pudieron llevarse a cabo debido a la inexistencia de un Banco Central independiente.

La devaluación fiscal fue una iniciativa innovadora que no llegó a realizarse, los efectos resultantes en países europeos permiten concluir que no difieren mucho de una devaluación del tipo de cambio: se incrementan las exportaciones, crece de manera momentánea el PIB, el empleo y se reduce el déficit de cuenta corriente.

En fin, un país dolarizado y expuesto a shocks externos, el cual, los últimos años ha financiado con deuda un elevado gasto público debería diseñar un ajuste fiscal que sea creíble y se pueda cumplir. Tomando en cuenta consideraciones especiales acerca del Ecuador los beneficios son al menos dos: por un lado, se incrementa la credibilidad de gobernante acerca del manejo responsable de la economía y por otro lado se asegura un aumento en el crecimiento económico a largo plazo debido a una mejora en las expectativas sobre la futura estabilidad macroeconómica.

\section{Referencias}

Afonso, A., Schuknetch, L., \& Tanzi, V. (2005). Public Sector Efficiency: Evidence for new EU Members States and Emerging Markets. European Central Bank Working Paper Series, 581. Recuperado de https://www.ecb.europa.eu/ pub/pdf/scpwps/ecbwp581.pdf?2c269a35246a4084e4df9e077ede9bc1

Armendáriz, E., \& Eduardo, C. (2016). La eficiencia del gasto de inversión pública en América Latina. XXI Congreso Internacional del CLAD sobre la Reforma del Estado y de la Administración Pública, Santiago, Chile, 8-11 noviembre de 2016. Recuperado de http://www2.congreso.gob.pe/sicr/cendocbib/ con4_uibd.nsf/3BOBFEE130669F58052580B200813641/\$FILE/armendar.pdf Banco Central del Ecuador. (2018). Información Estadística Mensual, Boletín 1993, Marzo 2018. Recuperado de https://contenido.bce.fin.ec/home1/ estadisticas/bolmensual/IEMensual.jsp

Barrios, S., \& Schaechter, A. (2009). Gauging by numbers: A first attempt to measure the quality of public finances in the EU. European Commission, Economic Papers, 382. Recuperado de http://ec.europa.eu/economy_finance/ publications/pages/publication15781_en.pdf

Blanchard, O. (1990). Suggestions for a New Set of Fiscal Indicators. OECD Economics Department Working Papers, 79, OECD Publishing, Paris. DOl: http://dx.doi.org/10.1787/435618162862.

Boscá, J., Doménech, R., \& Ferri, J. (2013). Fiscal Devaluations in EMU. BBVA Research Working Papers, 12 (11). Recuperado de https://www.bbvaresearch.com/KETD/fbin/mult/WP_1211_tcm348-338126.pdf

Contraloría General del Estado. (2018). Informe DNA3-0007-2018. Recuperado de $h$ ttp://Www. contraloria.gob.ec/WFDescarga. aspx?id=52076\&tipo=inf Fondo Monetario Internacional. (2014). Manual de Estadísticas de Finanzas Públicas. Recuperado de https://www.imf.org/external/Pubs/FT/GFS/ Manual/2014/GFSM_2014_SPA.pdf

Minsterio de Economía y Finanzas (2018). Boletín Deuda Externa Nro. 253, Enero 2018. Recuperado de http://www.finanzas.gob.ec/wp-content/uploads/downloads/2018/02/DE_ENERO-2018.pdf

Ribeiro, M. (2008). Eficiencia do gasto publico na America Latina: uma analise comparativa a partir do modelo semi-paramétrico com estimativa em dois estágios. Gestión Pública, 67. Recuperado de https://repositorio.cepal.org/bitstream/handle/11362/7329/1/S0800275_pt.pdf

Standard \& Poor's Financial Services. (2018). Listado de Calificaciones por Emisor América Latina de SP Global Ratings. Recuperado de https:// www. standardandpoors.com/es_LA/delegate/getPDF?article/d=2032759\&type $=$ COMMENTS\&subType $=$ REGULATORY

Végh, C., Vuletin, G., Riera-Crichton, D., Friedheim, D., Morano, L., \& Camarena, J. A. (2018). "Fiscal Adjustment in Latin America and the Caribbean: Short-Run Pain, Long-Run Gain? LAC Semiannual Report (Abril), World Bank Publications, The World Bank Group. Recuperado de https://openknowledge. worldbank.org/bitstream/handle/10986/29666/211290SP.pdf?sequence=6\&isAllowed $=y$ 